\title{
Diagnostics of cattle leucosis by using a biosensor based on surface plasmon resonance phenomenon
}

\author{
Z.S. Klestova ${ }^{1}$, A.Yu. Yuschenko' ${ }^{1}$, Yu.Yu. Dremukh ${ }^{1}$, O.F. Blotska ${ }^{1}$, E.F. Venger ${ }^{2}$, G.V. Dorozinsky ${ }^{2}$, \\ S.O. Kravchenko' ${ }^{2}$ Yu.V. Ushenin', N.V. Kachur², V.P. Maslov² \\ ${ }^{1}$ State Scientific-Control Institute for Biotechnology and Strains of Microorganisms \\ 30, Donetska str., 03151 Kyiv, Ukraine \\ E-mail: zinaklestova@gmail.com, \\ ${ }^{2}$ V. Lashkaryov Institute of Semiconductor Physics, NAS of Ukraine \\ 41, prospect Nauky, 03680 Kyiv, Ukraine \\ E-mail: gvdorozinsky@ukr.net,vpmaslov@ukr.net
}

\begin{abstract}
The virus of cattle enzootic leucosis leads to high losses in rural economy, implies forced slaughter of sick animals, loss of the breed, decrease in productivity, violation of reproduction processes in industrial breeding and livestock sector. Among cattle infection diseases, leucosis takes the leading position and comprises $57 \%$ of all the other nosological forms, if taking into account severity of injuries in organs, large-scale character of these diseases and economic aftermath. This disease can be transferred from animals to men faring with infected milk. The existing methods for cattle vital diagnostics are long-term and weakly-sensitive (AGID), or very complex and expensive (ELISA and PCR). In this work the alternative method for diagnostics is the method based on the SPR phenomenon was proposed. It has been shown for the first time that the SPR method enables to detect antibodies to cattle leucosis virus in the diluted solution (1 vol.\%) of weakly positive blood serum taken from sick animals, which cannot be made using the methods AGID and ELISA - in this case the serum is considered as negative, and the tested animal is considered as healthy, although it is carrier of virus.
\end{abstract}

Keywords: bovine leucosis, diagnostic test, surface plasmon resonance.

doi: https://doi.org/10.15407/spqeo22.01.111

PACS 73.20.Mf

Manuscript received 01.02.19; revised version received 14.02.19; accepted for publication 20.02.19; published online 30.03.19.

\section{Introduction}

Express testing the spread of retroviral infections among peoples and animals is an important world problem [1]. As known, retroviruses are causative agents for human secondary immunodeficiency that is observed as AIDS diseases, they are also the causative agents of the most dangerous viral diseases that are built-in to the cell genome, which is inherent, for example, to cattle enzootic leucosis [2]. It is noteworthy that these diseases can be transferred from animals to people, for instance, through use of milk infected with this virus [3]. Numerous publications and official veterinary statistical data show that, among cattle infection diseases, leucosis fully dominates, if taking into account severity of organ injuries, mass manifestation and economical losses, and comprises $57 \%$ as compared with other nosologies [4].

Retroviral infections affect mammals, birds, fishes. Especially spread is cattle enzootic leucosis that is registered in all the continents and many countries.
Prophylaxis and elimination of leucosis is very complicated because of its extremely wide spread. The special feature of this disease is the long-term incubation period that lasts without visible abnormalities in health condition, and this disease has various clinical presentations. Cattle leucosis virus (CLV) causes considerable economical losses in agriculture, it forces to kill sick animals, which leads to losses of breed, lowering the productivity, breakdown in processes of reproduction in animal breeding and livestock farming $[5,6]$.

Up to date, there developed are 20 different methods for lab diagnostics of leucosis, namely: haematological, histological and immunological ones that are based on detection of antibodies induced by viruses in blood serum of animals. To determine the status of CLV, the most often used are serological tests [7, 8]. The main method for intravital diagnostics of CLV is the reaction of immunodiffusion (AGID) as well as immuneenzyme analysis (ELISA). Besides, ELISA is used in problem-free herds of cattle to test the combined trials of 
milk from a group of animals [9]. To investigate especially valuable animals, there applied is the polymerase-chain reaction (PCR).

Both methods (AGID and ELISA) are widely available and relatively cheap ( $\$ 3-\$ 6$ per one test). They are based on detection of antibodies to viruses. The deficiency of these methods is their low sensitivity, therefore, detection of antibodies in animal's organism becomes available only for 6-14 weeks after infecting. Besides, these methods are relatively long (AGID 72 hours, ELISA - 2 hours) and sometimes give falsepositive results of serological tests for the cattle infected with CLV and non-infected one. As an alternative, one can use PCR to detect RNA of CLV, which provides some advantages over the serological methods. Application of PCR gives the possibility for early diagnostics of this infection, however, not all the PCR methods operate in a similar way, since they have definite differences in their sensitivity $(0.627-0.984)$ and specificity (0.89-1.0).

And since this virus can mutate, it is necessary to choose an individual PCR method for each case of diagnostics, which is an essential lack. Performing this test needs, as a rule, 8 hours, and its cost in Ukraine reaches $\$ 30-\$ 50$ per test. Detection of virus' RNA instead of antibodies as well as potential capability to detect small concentrations of this virus enables to use this test for newborn calves, cows in calf and recently infected animals, when serological tests cannot operate yet. However, the current cost of these PCR tests is too expensive for wide application in veterinary practice. The diagnosis of leucosis is considered to be confirmed under availability of one of positive results: in serological investigations with AGID or by using the immuneenzyme analysis and PCR.

Thus, the known methods of intravital diagnostics of CLV are either long-lasting and low-sensitive (AGID) or complicated and expensive (IEA and PCR). The absence of specific therapy and prophylaxis define topicality and priority of the problem of cattle leucosis not only for veterinary medicine but for biology as a whole. Therefore, development of diagnostic methods enabling to detect diseases at their initial stages and in the shortest terms is an extremely important and urgent task.

One of these diagnostic methods is the method based on the phenomenon of surface plasmon resonance (SPR). As compared with the traditional methods, this one has the following weighty advantages: capability to study processes of molecular interaction in nano-sized layers in a real time scale, small volume of the sample of studied substance (less than $10 \mu \mathrm{L}$ ), absence of the necessity to use special markers or fluorescent lables for the studied substance (analyte) [10]. The devices based on SPR have biological sensors consisting of a sensitive element and physical transducer.

The sensitive element is a thin metal layer $(40 \ldots 60 \mathrm{~nm})$ on the surface of dielectric substrate. In the majority of SPR devices, this metal layer consists of gold due to its high chemical inertness. The biological sensor on the surface of sensitive element contains biologically active substance (receptor) that selectively reacts only with respective analyte via covalent bonding that is also named as specific interaction. But really in any biological reactions, non-specific interaction takes place, when there present are partial filling the surface of sensitive element and domination of physical adsorption over covalent bonding, which lowers both sensitivity and selectivity of SPR devices.

To minimize non-specific adsorption on the surface of sensitive element, usually applied is adsorption of functional groups to immobilize receptors complementary to them. The most successful methods to functionalize the gold surface are based on the conception of molecular self-assembling thyols or disulphide molecules [11]. Development of highly stable receptors [12] enabled to perform effective testing the medicinal preparations by using the SPR method both in the process of their production and usage. SPR devices are widely used in detection of bacteria and viruses, namely: Staphylococcus aureus [13, 14], Pichia pastoris [15], Leishmania [16], Mycobacterium tuberculosis [17], human adenovirus [18], Epstein-Barr virus [19], bird's flu virus H5N1 [20], dengue virus [21], hepatitis B and AIDS [22, 23].

In [24], the authors have demonstrated non-invasive diagnostics of CLV by using the immunosensor analysis with the SPR method. Application of this method to cow lactoserum enabled to detect antibodies to proteins $p 24$, pg51 of CLV, presence of which indicates development of the disease in animals. Interaction of antigens (the proteins $p 24, p g 51$ of $\mathrm{CLV}$ ) with specific to them antibodies is registered as changes in the resonance angle of SPR. The authors of this approach use the modification of SPR sensor construction with the sensitive element from gold layer without any functional coating, i.e., without special preparation of the metal surface in this sensitive element.

Non-uniformity of the metallic layer in the sensitive element causes only partial interaction over the total surface of its area as well as presence of non-specific adsorption on the surface of the element, which defines a negative effect both on sensitivity and reliability of results obtained using the SPR method. This nonuniformity was caused, first of all, by the method of gold layer deposition, namely, vacuum sputtering. Therefore, we performed additional investigations that showed the necessity of specific preparation of the metal layer surface in the sensitive element to improve adhesion (immobilization) of the receptor.

Being based on these investigations, we developed a new way to produce the sensitive elements of SPR sensors. This way implies the annealing of sensitive elements with the preliminary deposited gold layer of the thickness $50 \pm 2 \mathrm{~nm}$ at the temperatures $100 \ldots 140{ }^{\circ} \mathrm{C}$ for 10 to $40 \mathrm{~min}$ and following irradiation with UV light of the wavelength $205 \ldots 315 \mathrm{~nm}$ also for $10 \ldots 40 \mathrm{~min}$ [25]. It provided to reduce non-uniformity of the sensitive element surface as well as improve, in what follows, its functionalization and to reduce non-specific adsorption 
on its surface. Applied in practice are sensitive elements of SPR sensors, which are preliminarily functionalized surface [26]. Some deficiency of this technology is an additional loss of time for activation of the functionalized surface and immobilization of the receptor on it. In essence, these operations are only preparation procedures, since the result of diagnostics is determined analyzing observed interaction of the receptor with analyte. In our opinion, an optimal way to reduce the time spent on diagnostics is to combine these preparation procedures of functionalization, activation and immobilization of the receptor.

In this work, we performed investigations of the influence of functionalization applied to the gold surface of sensitive elements in SPR sensors as well as preliminary antigen immobilization on the sensitivity of sensors when diagnosing cattle enzootic leucosis.

\section{Experimental investigations}

Studying the efficiency of gold surface functionalization and its influence on the sensitivity of SPR sensors was made with the SPR device "Plasmon-6" (Fig. 1) developed in the V. Lashkaryov Institute of Semiconductor Physics, NAS of Ukraine [11].

With this aim, we deposited the receptor - cattle antigen on the metal surface of sensitive elements. Then, we analyzed interaction of the receptor with analyte (i.e., antibody to CLV) with account of the relative shift observed for the minimum of the SPR resonant characteristics.

To interpret the results of investigations, in addition, we carried out testing the blood serum specific to CLV with antibodies by using the AGID method in the State Scientific-Control Institute for Biotechnology and Strains of Microorganisms (SSCIBSM).

To perform the above investigations, we used the following materials and reagents: re-inoculated cell culture of follicular lymphoma prepared from the sheep embryonic kidney FLK-BLV (FLK-SBBL, FLK 50/100, FLK 71, depositary at the National Center for Microorganism Strains at SSCIBSM), cattle antigen (cryobank of cell cultures at SSCIBSM), cow blood

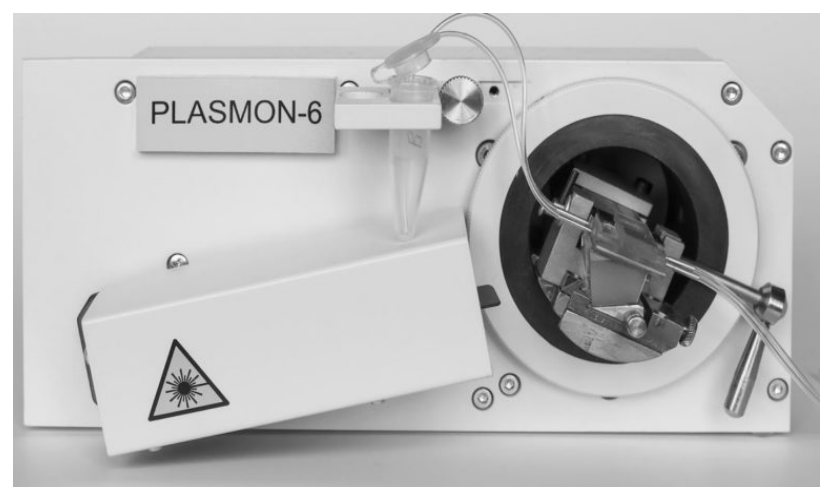

Fig. 1. External look of the device "Plasmon-6" with the SPR sensor. serum (husbandries of the Poltava region, Ukraine), nutrient medium "DMEM High Glucose", w/L Glutamine, w/o - Sodium Pyruvate, Sterile Filtered (Biowest, series S17885L0102, USA), cattle fetal blood serum "Fetal Bovine Serum Premium" (Biowest, series S14317S181B, USA), trypsin "Trypsin 0,25\%", w/o Calcium, w/o Magnesium, w/o Phenol Red, Sterile Filtered (Biowest, series S16067L0910, USA), 0.02\% versene solution (SSCIBSM), antibiotic "Gentamycin" (4\% solution for injections, pharmaceutic company "Zdorovya" Ltd, Ukraine) Triton X-100 (Merck, USA), 11-mercaptoundecan acid (Merck, USA), 11mercaptoundecanol (Merck, USA), absolute methanol ("Khimlaborreaktive" Ltd, Ukraine), N-ethyl-N'dimethyl-amine-propyl-carbo-diimide hydrochloride (EDC) (Merck, USA), N-hydrosuccinimide (NHS) (Merck, USA), physiological solution ("Pharmak" Ltd, Ukraine), twice distilled water (SSCIBSM).

Being aimed at unification and harmonization of methods for controlling the diagnostic ways in accord with international regulations and norms, developed in SSCIBSM was the National Standard for Leucosis Sera intended for standardization of ways targeted on serological diagnostics of enzootic CVL - "The set of blood sera for standardization of cattle leucosis virus antigen by using the reaction of immunediffusion (AGID)", registration certificate №BB-00664-06-13. With this aim, we received a set of cattle blood sera from the international reference lab studying enzootic bovine leucosis. This set is usually used as the reference one in the world practice to estimate quality of leucosis testsystems.

The re-inoculated culture of cells FLK was cultivated for 7 days in a special culture medium consisting of nutrient solution DMEM with addition of $10 \%$ cattle fetal blood serum preheated for $30 \mathrm{~min}$ at a temperature of $56{ }^{\circ} \mathrm{C}$. Replication of the leucosis virus was not accompanied by destruction of the cell monolayer, which corresponds to the main characteristics and norms of this culture. Then, supermatant liquid of the FLK cell culture that contains this virus was centrifuged and processed with de-ionized surface-active substance Triton X-100 to avoid adsorption of antigen on the walls of vessels. The concentrated and inactivated virus antigen was used to study its activity in AGID. With this aim, we prepared twofold dilutions of antigen $(1: 2 \ldots 1: 16)$, which were used with the standard blood sera. For measurements by using the SPR method and the device "Plasmon", we prepared dilutions of sera in physiological solution of $\mathrm{pH} 7.4$ that contained $0.14 \mathrm{M}$ of sodium chloride.

The sensitive elements of the device "Plasmon-6" were made in accord with the way described in [25]. To perform the experiments, we produced two groups of sensitive elements of five unities per each group with functionalized and non-functionalized metal surface. To provide functionalization, these sensitive elements were dipped one time into the mixture of following solutions: 11-mercapto-undecan acid $\left(0.88 \pm 0.01 \mathrm{mg} / \mathrm{cm}^{3}\right)$ in absolute methanol (20 vol.\%) and 11-mercapto- 
undecanol $\left(0.22 \pm 0.01 \mathrm{mg} / \mathrm{cm}^{3}\right)$ in absolute methanol (60 vol.\%) and were kept in an airproof glass vessel at the temperature $40 \pm 1^{\circ} \mathrm{C}$ for 6 hours.

After this keeping, the sensitive elements were first washed out in absolute methanol and then with distilled water by dipping them in Petri dish filled with these solutions by keeping there for $15 \mathrm{~min}$ in each solution. In what follows, the surface of sensitive elements was activated with the mixture of water solutions EDC and NHS by dipping them into this mixture in Petri dish and keeping there for $15 \mathrm{~min}$. Then, using a micropipette we coated the surface with $50 \mu \mathrm{L}$ of CLV antigen solution, kept at the temperature $20^{\circ} \mathrm{C}$ and later dried at the temperature $37^{\circ} \mathrm{C}$ for $20 \mathrm{~min}$. After that, the sensitive elements with immobilized and dried antigen were placed into the measuring double-channel cuvette inside "Plasmon-6" with the volume $5 \mu \mathrm{L}$ per channel. This cuvette was equipped with inlet and outlet fittings for each channel to remove studied liquids (distilled water and CLV antibodies). By turn, these liquids were pumped through one channel of the measuring cuvette with the peristaltic pump providing the velocity $10 \mu \mathrm{L} / \mathrm{min}$ for CLV antibody solutions and $50 \mu \mathrm{L} / \mathrm{min}$ - for buffer.

This velocity was chosen to minimize the pressure difference in the cuvette and provide a laminar liquid flow. This stage was followed by pumping the solution of antibodies through the cuvette to provide their binding with the receptor. The second channel was used as the reference one to compensate the influence of external factors, such as changes in the temperature of ambient medium. It was pumped through with the physiological solution. All the measurements were performed at the room temperature $\left(22 \pm 1^{\circ} \mathrm{C}\right)$.

To choose optimal duration suitable for immobilization of antigen-receptor on the functionalized surface of the sensitive element, we studied two cases: long-term immobilization (22 hours) and the mean-term one $(60 \mathrm{~min})$. The period of interaction between antigen and antibody was determined experimentally and equaled 36 min: this time was sufficient for binding of antigens with antibodies over the whole surface of the sensitive element in the SPR sensor, which was confirmed by the absence of any shift of the resonant characteristics after 30-min interaction (i.e., completion of the process). The processes of activation of the sensitive element surface (with EDC/NHS), of cattle antigen immobilization and interaction antigen-antibody were observed as respective angular shifts of the minimum inherent to the SPR resonance characteristics in time when pumping the necessary reagents through the measuring cuvette.

\section{Results}

To carry out the investigations by using the SPR method, we selected the samples of antigen that demonstrated a clear line of precipitation in AGID (Fig. 2). These samples were additionally cleaned using ultra-filtration and adsorption on porous glass with the pore diameter $100 \mathrm{~nm}$. In the experiments, we used 10-fold dilution of antigen in distilled water. All the sera were preliminary

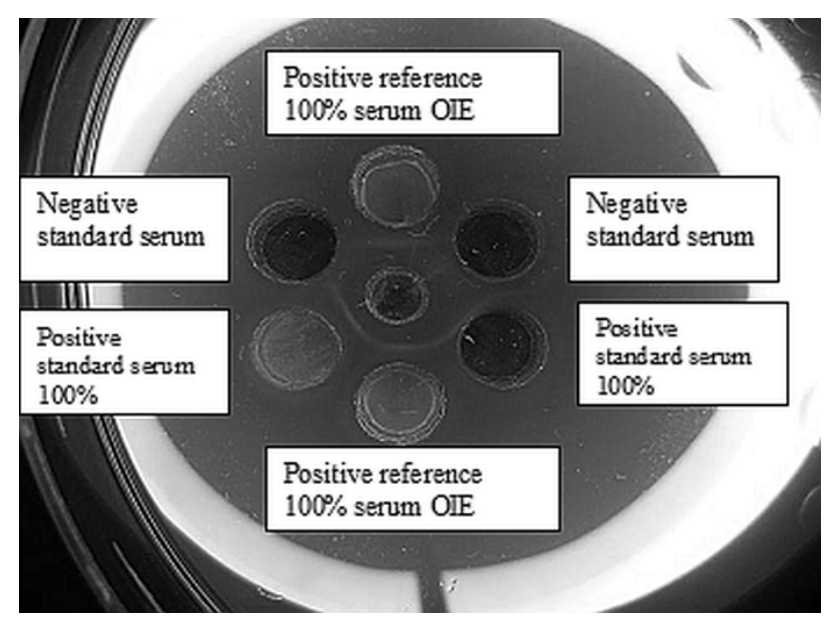

Fig. 2. Immunediffusion reaction of cattle antigen (central lunula) with the National standard samples of blood sera (positive $-100 \%$, weakly positive $-10 \%$, negative) and positive reference - peripherical lunulae.

tested in SSCIBSM and conditionally separated in three groups, namely: AGID-positive, AGID-negative and the doubtful ones, i.e., those non-providing in AGID a clear precipitation line in agar gel.

Long-term immobilization of antigen on functionalized and non-functionalized surfaces of sensitive element was carried out for 22 hours. When doing so, the antigen solution was cyclically pumped through the measuring cuvette. If studying interaction "antigen-antibody", we additionally used the weakly positive 10-fold diluted serum (10 vol.\%), since the 100 fold diluted serum could provide a signal close to the level of noise.

Fig. 3 shows the plot of kinetics corresponding to interaction "antigen - antibody" for two solutions of weakly positive sera ( 1 vol.\% and $10 \mathrm{vol} . \%$ ) in the process of long-term antigen immobilization on the surface of sensitive element with the functional coating and without it. The ordinate axis represents values of angular shifts of the SPR resonance characteristic minimum in angular degrees. As seen from the figure, when substituting biomaterials in the cuvette channels, one can observe the respective angular shift of the resonance minimum as a result of changing the refraction index at the surface of SPR sensor sensitive element.

The kinetics of surface activation and antigen immobilization are not shown in this figure, since these processes were not measured through their considerable total duration. The measurements started from washing out the cuvette channels with physiological solution (the tenth minute of kinetics). At the beginning of $25^{\text {th }}$ minute, this physiological solution was substituted with $1-\%$ serum solution that was pumped through for $100 \mathrm{~min}$ to reach saturation $\left(110^{\text {th }} \mathrm{min}\right)$. Then, the measuring channels were again washed out with the physiological solution. Starting from the $160^{\text {th }}$ min of kinetics, the cuvette was filled with the higher concentration (10 vol.\%). Like to the previous case, the serum was pumped through for $100 \mathrm{~min}$, and then the cuvette was washed out with the physiological solution. 


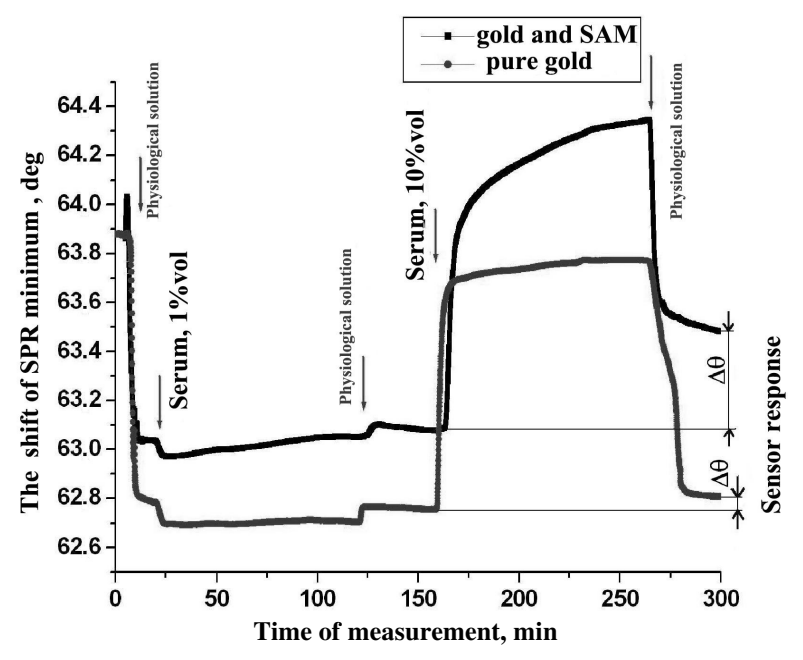

Fig. 3. Kinetics of interaction "antigen-antibody" for two solutions of weakly positive sera (1 and 10 vol.\%) after longterm ( 22 hours) immobilization of antigen on the surface of sensitive element with the functional coating and without it.

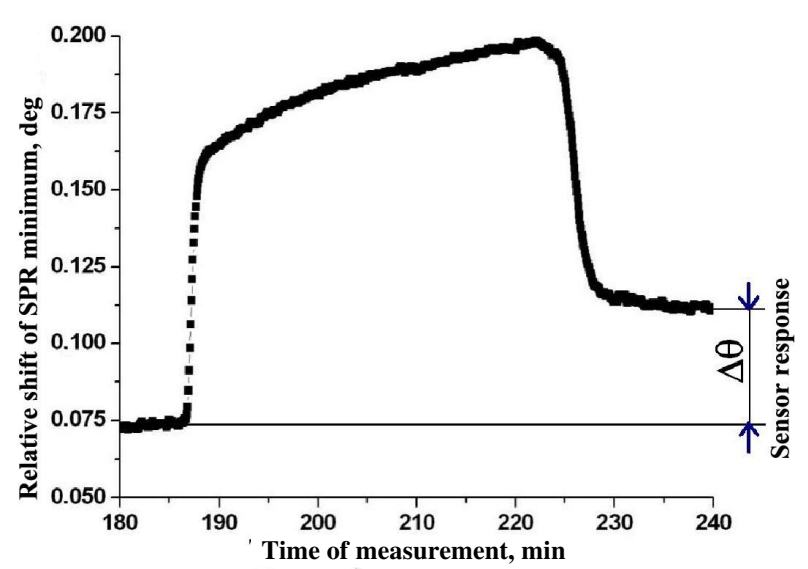

Fig. 4. Kinetics of interaction "antigen-antibody" on the surface of functionalized sensitive element for mean-time antigen immobilization $(60 \mathrm{~min})$ of the weakly positive serum with antibody in solution 100-fold diluted (Serum 1\%) in the physiological solution. The SPR sensor response equals $\Delta \theta=144 \pm 3$ arcsecond

In both cases of concentration, the physiological solution could not wash out the antibody in full volume, which indicated the presence of interaction, i.e., covalent binding between antigen and antibody. In the case of sensitive element without functional coating, the sensor response was considerably lower. It is related with the fact that the majority of antigen-receptors on the surface occupied wrong space position, and their sites for interaction with antibody were used for interactions "antigen-antigen" as well as "antigen-alkanthiol".

The angular shift of resonant characteristic for the sensitive element with the functional coating (Fig. 4) in the case of weakly positive serum with antibody, which was 100-fold diluted (1 vol.\%), was 9-fold higher ( $\Delta \theta=155 \pm 3$ arcsecond) than for the sensitive element without any functional coating $(\Delta \theta=17 \pm 3$ arcsecond). In the case of higher concentration of the 10-fold diluted weakly positive serum (10 vol.\%), the angular shift of resonance characteristic for the sensitive element with the functional coating was 8-fold higher ( $\Delta \theta=1454 \pm 3$ arcsecond) than that for the sensitive element without functional coating, which was $\Delta \theta=187 \pm 3$ arcsecond.

Shown in Fig. 4 is the kinetic of the SPR sensor response to the reaction of interaction "antigenantibody" on the surface of functionalized sensitive element for mean-time duration of antigen immobilization. The ordinate axis represents a relative shift of resonant characteristic minimum, which is the difference between signals in the measuring and reference channels. It provides compensation of the temperature drift of the resonant characteristic minimum that could occur, since the temperature factor has an essential influence on results of measurements [27]. For instance, the change in distilled water temperature by $0.25{ }^{\circ} \mathrm{C}$ results in the change of its refractive index by the value $2 \cdot 10^{-5}$, which is commensurate by its value with the detection limit of the device "Plasmon-6" $\left(2 \cdot 10^{-5}\right)$.

The procedures of washing out, activation, repeated washing and antigen immobilization with the following washing out in the physiological solution are not shown in this plot, since the increase in sensitivity is only determined with account of responses to interaction "antigen-antibody". It is seen from this plot that after the $187^{\text {th }}$ min substitution of the physiological solution by that of serum with antibody begins, and after the $190^{\text {th }}$ min there begins the proper reaction of covalent binding between antigen and antibody.

Starting from the $224^{\text {th }}$ min, we made washing out the cuvette with the physiological solution. As a result, the antibodies that were not bound with antigens were washed out, which was confirmed by the decrease in the relative shift of the resonant characteristic minimum to the value higher than the initial one inherent to the $180^{\text {th }}$ min of measurements.

When immobilization lasted for $60 \mathrm{~min}$, in the case of weakly positive serum with antibody in 100-fold dilution (1 vol.\%), the angular shift of resonant characteristic inherent to the sensitive element with functional coating was $\Delta \theta=144 \pm 3$ arcsecond, and for the element without this coating it was $\Delta \theta=18 \pm 3$ arcsecond.

Thus, when antigen immobilization lasts one hour, availability of the functional coating enhanced the sensitivity of the SPR sensor by 8 times, as compared with the sensitive element without this coating.

\section{Discussion}

Despite the long-term immobilization of the sensitive element surface without functional coating (Fig. 3), which provided filling all its surface, the response was relatively low $(\Delta \theta=17 \pm 3$ arcsecond). It can be explained as follows: the majority of receptors (near $80 \%$ ) took up such a position in space that their interaction with antibodies became impossible. 


\section{Conclusions}

The virus of cattle enzootic leucosis leads to high losses in rural economy, implies forced slaughter of sick animals, loss of the breed, decrease in productivity, violation of reproduction processes in industrial breeding and livestock sector. Among cattle infection diseases, leucosis takes the leading position and comprises $57 \%$ of all the other nosological forms, if taking into account severity of injuries in organs, large-scale character of these diseases and economic aftermath. This disease can be transferred from animals to men faring with infected milk. The existing methods for cattle vital diagnostics are long-term and weakly-sensitive (AGID), or very complex and expensive (ELISA and PCR).

The alternative method for diagnostics is the method based on the SPR phenomenon, but it is efficient and reliable only in the case of functionalization of the sensitive element surface in the SPR sensors. It has been demonstrated experimentally that application of this coating enhances the sensitivity of these sensors at least 8 -fold, as compared with the sensors without this coating. In this case, duration of diagnostics is only 4 hours and can be made shorter (for 2 hours) by respective optimization of this procedure. It has been shown for the first time that the SPR method enables to detect antibodies to cattle leucosis virus in the diluted solution (1 vol.\%) of weakly positive blood serum taken from sick animals, which cannot be made using the methods AGID and ELISA - in this case the serum is considered as negative, and the tested animal is considered as healthy, although it is carrier of virus.

\section{References}

1. Orliankin B., Guliukin M., Zamaraeva N., and Kunakov K. Taxonomy of retroviruses and characterization of bovine leukemia virus. Trudy Vserossiyskogo NII Eksperiment. Veterinarii im. Ya.R. Kovalenko. 1999. 72. P. 16-21 (in Russian).

2. Samuilenko A., Solovieva B., Nepoklonova E., and Voronina E. Infectious Pathology of Animals: in 2 vol. Moscow: Akademkniga, 2006 (in Russian)

3. Axel V.-C. Enzootic bovine leukosis and the risk to human health. African J. Biotechnol. 2017. 16, No 15. P. 763-770. DOI: 10.5897/AJB2016.15736.

4. Yarchuk B.M., Dombrovskii O.B, and Tyrsin R.V. et al. Leukemia of Cattle. Kyiv, Library of Veterinary Medicine, 2000 (in Ukrainian).

5. Nagaeva L., Aranchii S., Sinitsin V., Starodub M., Dobrosol G., and Nagaeva G. Diagnosis and prevention of leukemia of cattle. Kyiv, Library of Veterinary Medicine, 2003 (in Ukrainian).

6. Mammerickx M., Palm R., Portetelle D., and Burny A. Experimental transmission of enzootic bovine leukosis to sheep: Latency period of the tumoral disease. Leukemia. 1988. 2, No 2. P. 103-107.

7. Polat M., Takeshima S.N., and Aida Y. Epidemiology and genetic diversity of bovine leukemia virus. Virol. J. 2017. 14, No 1. P. 209225. DOI: $10.1186 / \mathrm{s} 12985-017-0876-4$.

8. Blotska O. Results of standardization and validation of diagnostic test-kits for Enzootic Bovine Leukosis. Vet. Biotechnol. Res. Bull. 2013. 23. P. 124-127.

9. World Organisation for Animal Health, 2.4.10. Enzootic Bovine Leukosis, in: Manual of Diagnostic Tests and Vaccines for Terrestrial Animals 2016. P. 721-732.

10. Dorozinsky G., Maslov V., and Ushenin Yu. Sensory Devices Based on Surface Plasmon Resonance. Kyiv, Politekhnika, 2016 (in Ukrainian).

11. Homola J. Present and future of surface plasmon resonance biosensors. Analytical and Bioanalytical Chemistry. 2003. 377, No 3. P. 528-539. DOI: 10.1007/s00216-003-2101-0.

12. Canovi M., Lucchetti J., Stravalaci M. et al. Applications of surface plasmon resonance (SPR) for the characterization of nanoparticles developed for biomedical purposes. Sensors (Basel). 2012. 12, No 12. P. 16420-16432. https://doi.org/10.3390/s121216420.

13. Kylväjä R., Kankainen M., Holm L., and Westerlund-Wikström B. Adhesive polypeptides of Staphylococcus aureus identified using a novel secretion library technique in Escherichia coli. BMC Microbiol. 2011. 11. P. 117.

DOI: 10.1186/1471-2180-11-117.

14. Mocan L., Ilie I., Matea C., Tabaran F., Kalman E., Iancu C., Mocan T. Surface plasmon resonanceinduced photoactivation of gold nanoparticles as bactericidal agents against methicillin-resistant Staphylococcus aureus. Int. J. Nanomedicine. 2014. 9, No 1. P. 1453-1461. DOI: 10.2147/IJN.S54950.

15. Shibui T., Bando K., and Misawa S. High-level secretory expression, purification, and characterization of an anti-human Her II monoclonal antibody, trastuzumab, in the methylotrophic yeast Pichia pastoris. Adv. Biosci. Biotechnol. 2013. 4, No 5. P. 640-646. DOI: 10.4236/abb.2013.45084.

16. Rodríguez P., Rojas H., Medina M. et al. Study of functionalized gold nanoparticles with Anti-gp63 IgG Antibody for the Detection of Glycoprotein gp63 in Membrane Surface of Leishmania Genus Parasites. Am. J. Anal. Chem. 2013. 4. P. 100-108. DOI: 10.4236/ajac.2013.47A014.

17. Hsu S.H., Lin Y.Y., Lu S.H., Tsai I.F., Lu Y.T., and Ho H.T. Mycobacterium tuberculosis DNA detection using surface plasmon resonance modulated by telecommunication wavelength. Sensors (Basel). 2013. 14, No 1. P. 458-467. DOI: 10.3390/s140100458.

18. Patent for useful model No. $46973 U$ (Ukraine). CI (2009) A61K 47/48, A61K 39/44. Immunosensory test system for the detection of antibodies against human adenoviruses in blood serum. N.V. Nesterova, L.M. Nosach, O.Yu. Povnytsia et al. N appl. u200907930, 27.07.2009. Publ. 11.01.2010. Bull. N 1. 
19. Khristosenko R., Nesterova N., Kostyukevych K. et al. Surface plasmon resonance immunosensor for detection of antibodies against the Epstein-Barr virus. Optoelectronics and Semiconductor Technics. 2011. 46. P. 92-99 (in Russian).

20. Bai H., Wang R., Hargis B., Lu H., and Li Y. A SPR aptasensor for detection of avian influenza virus H5N1. Sensors (Basel). 2012. 12, No 9. P. 12506-12518. DOI: 10.3390/s120912506.

21. Hu D., Fry S.R., Huang J.X. et al. Comparison of surface plasmon resonance, resonant waveguide grating biosensing and enzyme linked immunosorbent assay (ELISA) in the evaluation of a dengue virus immunoassay. Biosensors. 2013. 3, No 3. P. 297-311. DOI: 10.3390/bios3030297.

22. Heo N.S., Zheng S., Yang M.H. et al. Label-free electrochemical diagnosis of viral antigens with genetically engineered fusion protein. Sensors (Basel). 2012. 12, No 8. P. 10097-10108. DOI: 10.3390/s120810097.

23. Christopeit T., Øverb K., Danielson U.H., and Nilsen I.W. Efficient screening of marine extracts for protease inhibitors by combining FRET based activity assays and surface plasmon resonance spectroscopy based binding assays. Mar. Drugs. 2013. 11, No 11. P. 4279-4293.

DOI: $10.3390 / \mathrm{md} 11114279$.

24. Patent UA 81045 C2 (Ukraine).CI (2007) A61B 5/00, G01N 33/553 (2006.01), C12Q 1/70. Method of non-invasive diagnostics of leucosis in cattle by detecting antibodies to a virus in milk of cows with an immunosensor of the surface plasmon resonance. M.F. Starodub, V.P. Artiukh, L.B. Pirogova et al. N appl. a2005 12689, 28.12.2007. Publ. 26.12.2005.

25. Patent UA 111270 C2 (Ukraine) CI (2007) G01N 33/553 (2006.01). Method of diagnostics of cattle leucosis. E. Venger, V. Maslov, Yu. Ushenin et al. N appl. a2014 10363, 22.09.2014. Publ. 11.04.2016. Bull. N 7.

26. Homola J. Surface Plasmon Resonance Based Sensors. Springer-Verlag, 2006.

27. Özdemir S.K. and Turhan-Sayan G. Temperature effects on surface plasmon resonance: Design considerations for an optical temperature sensor. $J$. Lightwave Technol. 2003. 21, No 3. P. 805-814.

\section{Authors and CV}

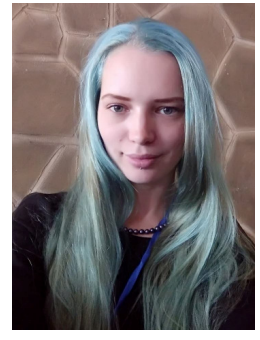

Yuschenko Alla - young scientist, biologist of the 1st category. She works at the Department of Biotechnology and Quality Controlling of Viral Preparations of SSCIBSM. She graduated from Taras Shevchenko Kyiv National University in 2014. In SSCIBSM, she works since 2016.

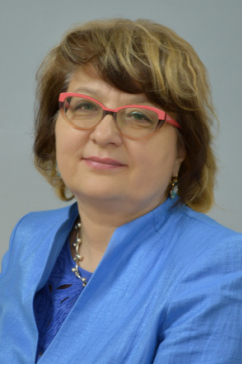

Prof., Dr. Zinaida Klestova

In 1981, graduated from Taras Schevchenko National Kiev University, Biology Faculty, specialty in genetic and virology. She possesses the academic degree of the Doctor of Veterinary Sciences and PhD of Biological Sciences and the academic status of the senior researcher (1993). At the State Scien-

tific-Control Institute of Biotechnology and Strains of Microorganisms, she is a Deputy Director of Scientific Work and the Head of scientific coordination of biosafety and biorisks Department. She possesses considerable experience of the research work and leading scientist in the spheres of biosecurity, biorisks, virology, microbiology, bacteriology, immunology and genetics.

E-mail: zinaklestova@gmail.com

ORCID: https://orcid.org/0000-0003-0771-7808

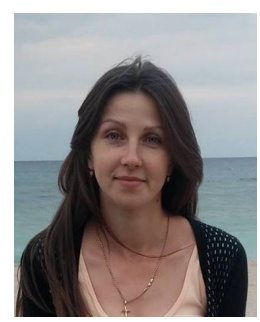

Dremuh Julia,

Ph.D. student. Education graduated from Veterinary college in 2008 by the specialty Veterinary Medicine. In 2008, she entered the higher education institution National University of Life and Environmental Sciences of Ukraine and attained complete higher education by the specialty Veterinary Medicine. In 2012, Dremuch entered a $\mathrm{PhD}$ program at the Institute of Veterinary Medicine of NAAS by the specialty 16.00.03 - Veterinary Microbiology, epizootiology, infectious diseases and immunology. She works at the laboratory of virology and antiviral drugs.

E-mail:dremych@gmail.com

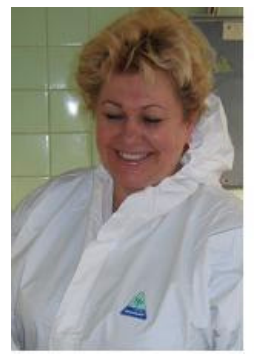

Oksana Fedorivna Blotska DVM, PhD, Head of the Division of Check and Industrial Virus Strains Support of State Scientific Control Institute of Biotechnology and Strains of Microorganisms, Kyiv, Ukraine. She possesses considerable experience of the research work and is a skilled scientist in the spheres of virology, microbiology, immunolo-

gy, serology, biosafety, biosecurity and biorisks. She was the supervisor of 2 State budget 3-years scientific programs, manager of 2 and member of one International Projects. She has published 139 scientific articles, 3 patents pending, created 7 new immunobiological means and 8 new National standards. E-mail:blotskaya@ua.fm 


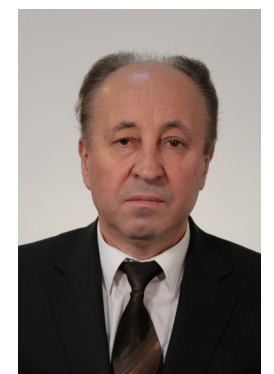

Dr. Yevgen Venger, Head of the scientific department, Corresponding Member of NAS of Ukraine, Dr of Sciences, Professor. He has an experience of managing the State scientific and technical program for development of sensor devices in 2008 - 2017, projects by the State order and innovation ones.

E-mail: vengeref@gmail.com

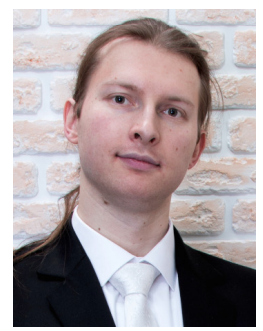

Glib Dorozinsky got his degree MS in Physics and Techniques (Electronic home appliances) at the National Technical University of Ukraine "Kiev Polytechnic Institute", Radiotechnical faculty (Ukraine) in 2012. Since $2016 \mathrm{PhD}$, senior researcher at the V. Lashkaryov Institute of Semiconductor Physics, NAS of Ukraine.

His research interests lie in physics of surfaces, development and design chemical sensors and biosensors for applying in different fields like medicine, pharmacology, industry and ecology.

E-mail: gvdorozinsky@ukr.net

ORCID: https://orcid.org/0000-0002-7881-2493

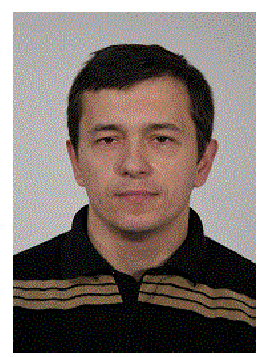

Serhii Kravchenko, Senior researcher, $\mathrm{PhD}$.

Graduated from Lviv National Technical University, specializing in technology engineer, chemical technology of biologically active compounds. Graduated from the Institute of Bioorganic Chemistry of the National Academy of Sciences of Ukraine. Since 2006, he works at

the V. Lashkaryov Institute of Semiconductor Physics NAS of Ukraine, currently he holds the position of Senior Researcher. The field of scientific interests is the development of sensitive elements of physical converters (QCM, SPR, SERS), obtaining plasmon and fluorescent nanoparticles for sensory applications.

E-mail: kravchenko.srg@gmail.com

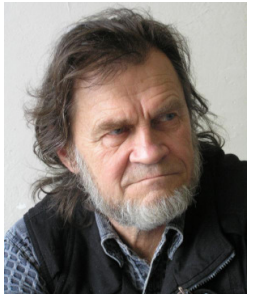

Yurii Ushenin got his degree in Vision and spectroscopy at Taras Shevchenko National University of Kyiv. He graduated from the Kiev University, Physics department (Optical instruments and spectroscopy) in 1971. Since 1973 he was with the Institute of Semiconductor

Physics of National Academy of Sciences of Ukraine as an engineer and a senior researcher (since 1992 up to now). His areas of expertise include systems of collection and data processing, software design (Pascal, Delphi) and optical devices including SPR and planar semiconducting interferometer. E-mail: ushyury68@i.ua

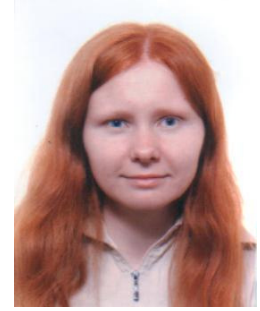

Nataliya Kachur got her degree MS in Physics and Techniques at the National Aviation University of Ukraine, Mechanical faculty (Ukraine) in 2006.

Her research interests in physics of surfaces, development and design of sensors for application in control of quality of transparent materials.

E-mail: natalykachur@gmail.com

ORCID: https://orcid.org/0000-0001-6868-8452

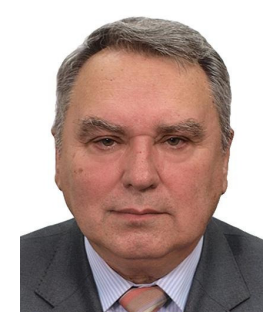

Dr. Volodymyr Maslov got his degree in Physics and Technics at the Moscow Institute of Physics and Technology State University (Russia) in 1969, his $\mathrm{PhD}$ in Material Science at the Moscow Institute of Physics and Technology State University (Russia) in 1973. Doctor of Material Science since 2009, Honored inventor of Ukraine.

$\mathrm{He}$ is professor of National Technical University of Ukraine "Kyiv Polytechnic Institute" since 2010. His research interests in several topics of optical engineering and physical behavior of functional materials and phenomena of surface plasmon resonance and application of it in medicine and ecology now.

E-mail: vpmaslov@ukr.net

ORCID: https://orcid.org/0000-0001-7795-6156 